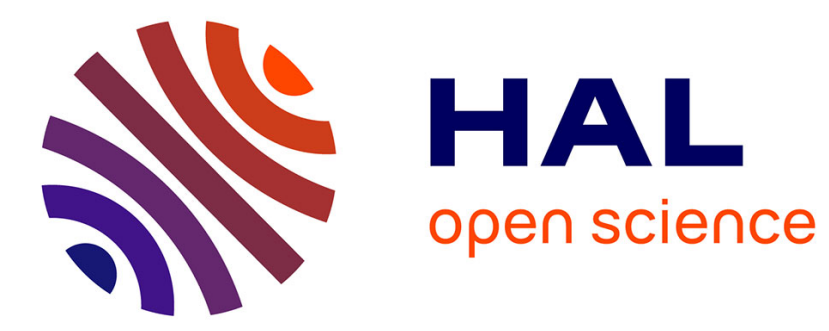

\title{
Vivien chez Mabile dans les Enfances Vivien : une fenêtre sur l'avenir
}

\author{
Adeline Richard-Duperray
}

\section{To cite this version:}

Adeline Richard-Duperray. Vivien chez Mabile dans les Enfances Vivien: une fenêtre sur l'avenir.

Par la fenestre, 2003. hal-01699833

\section{HAL Id: hal-01699833 \\ https://hal.science/hal-01699833}

Submitted on 2 Feb 2018

HAL is a multi-disciplinary open access archive for the deposit and dissemination of scientific research documents, whether they are published or not. The documents may come from teaching and research institutions in France or abroad, or from public or private research centers.
L'archive ouverte pluridisciplinaire HAL, est destinée au dépôt et à la diffusion de documents scientifiques de niveau recherche, publiés ou non, émanant des établissements d'enseignement et de recherche français ou étrangers, des laboratoires publics ou privés. 


\title{
Vivien chez Mabile dans les Enfances Vivien: une fenêtre sur l'avenir.
}

\author{
RICHARD Adeline \\ (Université de Provence)
}

Si l'on imagine Vivien, bien souvent considéré comme un épigone de Roland, c'est sur le champ de bataille aride et désolé de Larchamp, ou mourant dans le décor symbolique d'une source édénique ${ }^{1}$. Ce n'est pas un personnage palatin. Et si on l'aperçoit près d'une fenêtre dans La Chanson de Guillaume, c'est que tout le peuple sarrasin attend dehors l'armée chrétienne pour le combat'. On mesure tout ce qui le sépare du Vivien enfant, élevé chez des marchands, regardant un paysage champêtre qui a tout du locus amoenus depuis la fenêtre de la demeure de ses parents adoptifs. Cependant, ce décalage n'est pas le fait le plus problématique de ce passage des Enfances Vivien. En effet, si dans la Chanson de Guillaume le rôle de la fenêtre se laisse facilement appréhender (elle sert à introduire le nœud de l'action), il n'en est pas de même ici. Certes, elle est une ouverture qui permet au personnage de regarder vers l'extérieur, mais sa présence même avec le paysage sur lequel elle donne laisse une impression curieuse et fait figure d'élément rapporté.

Dès lors, faut-il considérer cette fenêtre et la vue qu'elle révèle comme des réalités faisant partie de l'univers fictionnel dans lequel évolue notre héros, ou plutôt comme des métaphores d'un ailleurs qui ne se laisserait qu'apercevoir par l'ouverture nécessairement réduite d'une embrasure ? La fenêtre permet de voir audelà du présent de la fiction, dans le futur du personnage et du récit de ses aventures. Mais peut-être faut-il voir derrière elle une nouvelle fenêtre découvrant une vue réflexive de l'épopée sur elle-même.

Cette scène à la fenêtre se présente avant tout comme un tableau. Si le personnage est montré dans cette attitude, c'est pour mieux être offert à l'imagination du lecteur. Il fait en effet l'objet dans cette laisse d'une assez longue description, qui insiste à la fois sur sa grâce, sa jeunesse voire sa fragilité ${ }^{3}$ :

\footnotetext{
${ }^{1}$ La source auprès de laquelle meurt Vivien est une apparition surprenante qui contraste avec le reste de la plaine de Larchamp, présentée comme désertique.

${ }^{2}$ La Chanson de Guillaume, éditee par François Suard, Paris, Bordas (collection Classiques Garnier), 1991, laisses IX et X.

${ }^{3}$ Jusqu'alors, Vivien a en effet été présenté comme un tout jeune enfant (il a sept ans, comme on le voit aux vers 47-49 du manuscrit Paris, B.N., fr. 1448) qui, quoique très courageux, est encore assez vulnérable pour demander à sa mère de le prendre dans ses bras lorsqu'il se sait en danger (cf. v. 491-494, Boulogne-sur-Mer, Bibliothèque municipale 192, vv. 889). La délicatesse du personnage peut alors suggérer aussi une certaine fragilité.
} 


\begin{abstract}
lou chief ot blonde menu recercele les iols ot vars comme faucons mue blanche ot la char comme flor en este ce fut li anfes qui puis soffrit maint mel car il conquist les archans sor la mer (Paris, B.N., fr. 1448, vv.888-892).
\end{abstract}

C'est un enfant vulnérable, exilé loin des siens dans un monde qui lui est inconnu, celui des marchands.

Ce portrait, suivi aussitôt de la description du paysage qui s'offre par la fenêtre, introduit ainsi dans l'action une pause parallèle à celle que constitue la vie marchande du personnage dans sa vie chevaleresque: il est destiné à combattre car fils de chevalier, mais condamné à l'inaction car il est le fils adoptif de parents bourgeois. Ce temps de répit permet au pathétique de se développer et d'amener sans heurt le passage à l'évocation nostalgique de sa parenté par le héros jusque-là pris dans l'action.

Cette fenêtre s'oppose à d'autres fenêtres épiques auxquelles nous avait habitués la geste de Guillaume d'Orange où elles donnent à voir l'imminence du combat : ennemis à affronter, amis à secourir ${ }^{4}$. Il n'en est rien dans les Enfances Vivien, nulle trace visible de danger passé, présent ou à venir; au contraire, le paysage est agréable et riant, c'est un locus amoenus, avec les topoi attendus :

\author{
il regarda tout contreval le pre \\ voit la riviere les rosiers boutonner \\ et liaue courre et les vignes delez \\ et oit la merle et lourieul chanter \\ (Londres, British Library, Royal 20 DXI, vv. 913-916).
}

La fenêtre est un espace circonscrit qui, par son étroitesse et son rôle de frontière, isole le héros de l'espace extérieur; mais aussi, parce que Vivien, tourné vers le paysage, se met à l'écart de ceux qui se trouvent à l'intérieur, elle l'isole de l'espace du dedans. Il est donc, d'un point de vue strictement spatial, un personnage marginal qui se tient seul dans un lieu de l'entre-deux ${ }^{5}$. Elle maintient le héros entre deux réalités, plus encore qu'une porte car, contrairement à cette dernière, la fenêtre ne permet pas un passage aisé. On s'y tient, à la fois dehors et dedans, prisonnier d'un lieu hors normes, sans pouvoir véritablement en sortir puisqu'on ne peut réellement accéder au dehors. Le seul mouvement possible est une régression vers l'intérieur. N'étant plus considéré comme un chevalier sans être non plus un bourgeois, Vivien

\footnotetext{
${ }^{4}$ Nous renvoyons ici entre autres aux laisses déjà citées (p. 1) de la Chanson de Guillaume.

${ }^{5}$ Il faut cependant remarquer qu'il existe un manuscrit (Paris, B.N., fr. 24369, v. 149-177) où la scène à la fenêtre ne fonctionne pas de la même manière, puisque la dispute engagée entre Vivien et son père adoptif se poursuit alors que le jeune homme est en train de contempler le paysage. La suspension de l'action est bien un choix délibéré dans les manuscrits que nous étudions.
} 
se trouve dans un entre-deux social, attiré par le monde qu'il a quitté mais retenu chez les marchands, exilé mais incapable de pallier la recreantise qui le menace.

Cette étrangeté dans la disposition est redoublée par le fait que le surgissement de la fenêtre dans le texte est aussi brutal qu'inattendu. En effet, rien n'indique au départ que l'on se trouve dans un endroit fermé : les retrouvailles avec Godefroy se font dans un lieu indéterminé. Pour preuve de ce manque de précision, le manuscrit Paris, B.N. fr. 1448 remplace la fenêtre par une fontaine, lieu d'extérieur selon toute probabilité6. De même, s'il est dit que les marchands observent Vivien, on ne sait pas où ils se trouvent, s'ils l'aperçoivent du dehors par la fenêtre ou s'ils se trouvent à côté de lui :

cil marcheant sen sont garde done dist luns a lautre onques ne matendes sonkes cis fu de marchant engendres

(Boulogne-sur-Mer, Bibliothèque municipale 192, vv. 929-931)

On est dans un espace intermédiaire, dans un entre-deux qui semble ne plus répondre aux critères spatiaux habituels. Mais peut-être faut-il comprendre simplement que cette fenêtre n'est pas un lieu réel mais un lieu abstrait, métaphorique, une fenêtre à fonction symbolique.

Elle peut être en effet appréhendée comme la représentation d'une ouverture sur le futur d'un héros en devenir. Le récit d'enfance en lui-même est en quelque sorte une fenêtre sur l'avenir, mise en abyme dans le passage que nous étudions. Il ne s'agit pas de n'importe quel récit d'enfance mais, puisqu'il a été écrit a posteriori de La Chanson de Guillaume, d'Aliscans ${ }^{7}$, voire de La Chevalerie Vivien, on sait que le petit Vivien est destiné à devenir l'un des plus grands héros de la lutte française contre les Sarrasins. La fenêtre se présente donc ici comme une brèche dans le présent, par laquelle on peut apercevoir le futur guerrier d'exception ${ }^{8}$. C'est pourquoi la scène de la fenêtre est précédée par le portrait de Vivien déjà cité, destiné à prouver que ce dernier est l'archétype du jeune chevalier, élément nécessaire dans une oeuvre selon laquelle un membre d'une haute lignée ne peut se défaire des traits et des valeurs caractéristiques de sa classe sociale. Dès lors, cette fenêtre est pour Vivien une vue sur sa véritable nature et lui apporte la révélation sur son incapacité à changer pour devenir le véritable fils de ses parents adoptifs.

\footnotetext{
${ }^{6}$ «Mais Wiviens ne sest mie arestes/ en sa main porte un esprevier mue/ a la fontaine san vait por deporter $\gg$ (v. 909-911).

${ }^{7}$ Aliscans, tome I et II, publié par Claude Régnier, Paris, Champion, 1990.

${ }^{8}$ C'est en effet le souvenir de son lignage chevaleresque que réactive la présence de la fenêtre, comme nous allons l'étudier plus loin : «lors li remambre de son fier parente/ de son aioul li prent a remanbrer/ et de girart et dernalt lou sane/ et de guarin de guichart lalose/ et de guillaume lou marchis au corneis »(v. 917-921, Paris, B.N. fr. 1448).
} 
En outre, cette scène à la fenêtre est aussi le théâtre d'un changement dans le personnage du jeune garçon. Le début de la chanson nous montre un Vivien bien éloigné du guerrier farouche qu'il est destiné à devenir, un petit enfant qui a encore besoin de la protection de sa famille et dont la fragilité incite à le prendre en pitié. $\mathrm{Si}$ cette caractéristique sert encore ici le pathétique de la scène, dans le cadre de l'évocation nostalgique de la famille perdue, il s'ajoute paradoxalement une notion de force et de maturité précoces dans la double évocation du faucon mue ${ }^{10}$ aux yeux duquel on compare ceux de Vivien et dans celle de l'esprevier mue ${ }^{l l}$ qu'il porte à son poing. Evidemment, ces oiseaux de chasse de très grand prix, réservés à la noblesse, sont là pour insister sur la haute extraction du jeune garçon. S'ils sont en quelque sorte des métonymies de Vivien, il faut remarquer que ce sont des oiseaux adultes, mues, donc déjà capables de chasser. Par ailleurs, la chasse au faucon ou à l'épervier demande une grande connaissance de cet art, dont il faut alors supposer que Vivien la possède déjà ou tout au moins veut la posséder. On sait aussi que la chasse n'est qu'une transposition de la guerre sur le mode ludique et qu'elle lui sert de substitut. D'ailleurs, quelques vers plus haut, Vivien a déjà exprimé deux désirs, finalement assez similaires, celui de pouvoir chasser à sa guise et celui d'aller au combat, en particulier dans la lutte contre les Sarrasins à l'instar de ceux de sa lignée. La fenêtre s'ouvre donc sur le futur et fier guerrier de Larchamp.

C'est d'ailleurs l'occasion pour les marchands de reconnaître en Vivien un Aymeride qu'ils suivront plus tard dans la guerre contre les Sarrasins. C'est non seulement la figure du héros combattant qui se dessine ici, mais encore celle du chef de guerre, capable de rallier ses troupes dans un combat désepéré où la mort leur est promise. Il est donc important qu'il se resitue à l'intérieur de son lignage héroïque pour rappeler au lecteur comme aux marchands ${ }^{12}$ son caractère de héros-chevalier, d'où l'importance que revêt la longue énumération de sa parenté dont le paysage le fait se souvenir. La valeur qu'il a héritée d'elle, il en fait immédiatement preuve dans ses sentiments. A l'évocation de sa famille absente, au regret d'être rejeté en marge de ce lignage chevaleresque, il ne peut retenir ses larmes, et ce topos est la preuve de sa noblesse de coeur, la délicatesse des sentiments étant une qualité indispensable à une âme bien née. La rêverie nostalgique et la perméabilité à la puissance évocatrice d'un paysage n'est pas à la portée d'un coeur vil. La fenêtre est donc le catalyseur qui révèle définitivement la nature héroïque de Vivien ${ }^{13}$, à lui-même comme aux autres. Cela se manifeste d'ailleurs par le jeu des regards : celui de Vivien se tourne

\footnotetext{
${ }^{9}$ A l'occurrence que nous avons déjà citée, nous pouvons ajouter les vers 536-555 (Paris, B.N. fr. 1448) où Guillaume doit intervenir pour secourir son neveu maltraité par un Sarrasin.

${ }^{10}$ Boulogne-sur-Mer, Bibliothèque municipale 192, vv. 889.

${ }^{11}$ Boulogne-sur-Mer, Bibliothèque municipale 192, vv. 910.

${ }^{12}$ Ceux-ci semblent en effet l'avoir entendu : « cil marcheant san sont garde done » (Paris, B.N. fr. 1448 v. 929)

${ }^{13}$ Cette nature, comme on le perçoit déjà, est composite : Vivien se définit à la fois comme héros épique, romanesque et comme héros de fiction de manière plus générale. Nous allons revenir sur ce point dans notre étude.
} 
vers l'extérieur, vers ce qui offre de nouvelles possibilités, vers l'infini; dans le même temps, il est l'objet de tous les regards, admiratifs, des marchands. La fenêtre traduit ainsi à la fois la double capacité du héros à aller de l'avant, à voir l'ailleurs, et à attirer tous les regards, à être l'objet d'une adhésion et d'un désir quasi-unanime. Paradoxalement, la fenêtre est à la fois centrifuge et centripète, elle attire et retient le héros dans son entre-deux en même temps qu'elle le projette vers son futur par sa fonction prospective.

En révélant la nature héroïque de Vivien, la fenêtre ouvre sur tous les possibles du récit et représente un nouveau départ pour la fiction au moment où le récit avait apparemment abouti à une impasse avec «l'embourgeoisement» du héros. Devenir marchand, c'était renoncer à toute action guerrière et donc, pour un personnage épique, trouver la mort littéraire. Le récit est ici relancé.

Dans le manuscrit de Boulogne-sur-Mer, Bibliothèque municipale 192, ce rôle de la fenêtre est marqué du point de vue métrique par la présence d'un petit vers hexasyllabique. Même si ce n'est pas le cas le plus fréquent dans les chansons de geste, une laisse peut annoncer l'action développée par la suivante ${ }^{14}$. C'est ce qui se passe ici, où le vers hexasyllabique devient une sorte de fenêtre sur l'action; Vivien y prend la décision d'agir, en acceptant la marchandise de Godefroy avec cependant une arrière-pensée, inconnue du lecteur, mais qu'il ne peut qu'imaginer tournée vers des aspirations plus nobles que celles du profit:
a bone estrine li a cent fer done
tenes biax fiex de gainer penses
viviens les prent ne les vielt refuser
il seit bien que il pense
(Boulogne-sur-Mer, Bibliothèque municipale 192, vv. 942-945)

De même que le vers hexasyllabique de la laisse précédente annonçait la merveille qu'est ce Vivien, indéfectiblement noble au milieu des bourgeois ${ }^{15}$, la scène de la nostalgie à la fenêtre est une ouverture, voire un passage, vers la nouveauté: on regarde et même on bascule vers un avenir radicalement différent. Le vers hexasyllabique redouble du point de vue métrique le motif de la fenêtre.

Cet avenir, le lecteur averti le connaît déjà, il sait que le paysage aperçu de la fenêtre est trompeur et que celle-ci donne en réalité sur le rivage de Larchamp. Il est amené à voir une forme de préfiguration dans le portrait de Vivien. En outre, ce portrait est suivi de manière inopinée par l'annonce du martyre de Vivien à Larchamp ${ }^{16}$. Il existe une fragilité constitutive du personnage (qui ressort d'ailleurs

\footnotetext{
${ }^{14}$ On sait que le système d'écriture des chansons de geste fonctionne souvent selon le système du rappel : une laisse commence par résumer l'action de la laisse précédente.

15 «Godefrois lot toz li sans li remue/ molt en ot grant mervelle» (Boulogne-sur-Mer, Bibliothèque municipale 192, vv. 826-827).

${ }^{16}$ «Ce fut li anfes qui puis soffrit maint mel/ car il conquist les archans sor la mer » (Paris, B.N. fr.1448, vv. 891-892). Qui connait l'histoire de Vivien sait que les maux ici évoqués débouchent
} 
dans les descriptions qui sont faites de lui lors de son agonie dans les autres oeuvres ${ }^{17}$ ), destiné à mourir avant d'avoir vécu, à être une victime propitiatoire. Donc, s'il y a préfiguration, notre fenêtre, outre qu'elle est peut-être plus symbolique que réelle, n'est qu'une fausse ouverture, puisque depuis la Chanson de Guillaume, le destin de Vivien est scellé. L'ouverture qu'elle propose est ailleurs, dans un avenir littéraire où rien n'est jamais joué et tout reste toujours à réinventer.

C'est pourquoi Vivien nous est montré en train de chanter tandis que des oiseaux lui répondent en écho :

\author{
a la fenestre sen vet por deporter \\ molt doucement prist un son a chanter \\ il regarda tout contreval le pre [...] \\ et oit la merle et lourieul chanter \\ lors li ramembre de son grant parenté \\ (Londres, British Library, Royal 20 DXI,vv. 911-917).
}

On notera l'importance stylistique accordée au verbe chanter par la répétition, tout d'abord, mais aussi par la répétition à la rime, place stratégique qui fait résonner en écho et musicalement ces deux occurrences. Ce n'est que par l'entremise de ce chant que le paysage lui permet d'évoquer sa parenté, comme le prouve l'adverbe lors. Son chant replace Vivien dans une lignée de héros ; nouvel Orphée, c'est lui-même qu'il vient tirer des limbes que représente pour un Aymeride l'embourgeoisement ; trouvère, il chante son statut de héros guerrier et devient créateur de sa propre geste. De la geste des Aymerides dont il fait l'énumération, se détache, indépendante, celle de Vivien. Son chant et sa geste s'élèvent, solitaires, et trouvent une ouverture propre. En outre, c'est à présent sa voix qui évoque les autres noms célèbres de sa parenté ; il les convoque dans sa geste au lieu d'être convoqué dans celle des autres. L'auteur révèle qu'à l'intérieur du cycle de Guillaume d'Orange, il se trouve à présent une geste de Vivien.

En se détachant du restant du cycle, celle-ci va accentuer ses particularités, et même trouver une voie originale. C'est cette voie qu'indique la fenêtre ouverte, observatoire de notre personnage. Elle s'ouvre en effet sur le romanesque. L'épisode de Vivien chez Mabile au coeur duquel elle prend place est une enclave romanesque à l'intérieur de l'oeuvre épique que constituent les Enfances Vivien. La fenêtre est une sorte de mise en abyme de cet épisode : comme celui-ci est une échappée temporaire de l'épique vers le romanesque, la fenêtre est une échappée vers l'extérieur, vers un paysage que l'on peut lui-même qualifier de romanesque.

dans toutes les chansons sur la mort du personnage. C'est pourquoi nous sommes fondés ici à parler de martyre.

${ }^{17}$ Nous renvoyons ici aux récits de la mort de Vivien : laisses CXXXI à CXXXIII de La Chanson de Guillaume et surtout les laisses XIV et XXIV à XXVIII d'Aliscans. 
Dans cet épisode, on retrouve un topos caractéristique du roman, celui de la reverdie associée au chant et à la mélancolie ${ }^{18}$ :

\author{
il regarda tout contreval le pre \\ voit la riviere les rosiers boutonner \\ et liaue courre et les vignes delez \\ et oit la merle et lourieul chanter \\ (Londres, British Library, Royal 20 DXI, vv. 913-916)
}

En regardant ce paysage, Vivien regarde vers le romanesque, au propre comme au figuré. Sa réflexion catalysée par le décor est elle-même un topos romanesque ${ }^{19}$. Ce rôle du décor tranche nettement avec celui qui lui est dévolu dans le reste de la geste de Vivien ${ }^{20}$. Son traitement dans les Enfances Vivien est donc un fait marquant : il signale le moment où l'épique bascule vers le romanesque.

La fenêtre est une porte vers un autre genre. Cela est directement induit par le type du récit d'enfance :

En réalité, les récits d'enfances sont aussi voués à produire de nouveaux types de héros, qui font contraste avec le héros épique traditionnel. [...] L'apparition des enfances dans l'épopée en vers se situe donc dans la perspective du renouvellement du genre par la différenciation des personnages ${ }^{21}$.

Vivien a donc dû être doté d'une histoire plus complète et plus particulière que celle de son combat et de sa mort à Larchamp, qui suivaient si scrupuleusement la trame épique qu'on a longtemps considéré le héros de La Chanson de Guillaume et d'Aliscans comme un autre Roland, preuve que la «différenciation des

${ }^{18}$ Le plus souvent, il est vrai, la reverdie est associée au renouveau de l'aventure et de l'amour, mais elle peut aussi suggérer la nostalgie, par exemple lorsque l'on est éloigné de l'être aimé, comme c'est le cas pour Tristan dans Le Roman de Tristan en Prose, édité par Philippe Ménard, 9 volumes, Genève, Droz, 1987-1997, tome VI, $\S 158$. C'est avant tout un topos lyrique, qui a ensuite été largement réutilisé par le roman, qui se l'est approprié. L'épisode des enfances chez Mabile présentant de très nombreuses similitudes avec ce dernier genre, nous pensons que nous pouvons voir dans cette reverdie un motif à coloration romanesque.

${ }^{19}$ «Le thème du retour sur soi que favorise la contemplation d'un paysage agréable relève du romanesque » (Bernard GUIDOT: «Les «enfances» de Vivien ont-elles un caractère romanesque ? », dans PRIS-MA, bulletin de liaison de l'équipe de recherche sur la littérature d'imagination du Moyen Age, tome XII, n² 2 , juillet-décembre 1996, p. 172). On peut notamment se rappeler le célèbre incipit du Roman de la Rose.

${ }^{20}$ Presqu'absent de la Chevalerie Vivien (La Chevalerie Vivien, éditée par Duncan MAC MILLAN, tomes I et II, Senefiance ${ }^{\circ} 39$ et 40, Aix-en-Provence, éditions du CUER MA, 1997) il participe au tragique dans La Chanson de Guillaume par sa disposition en huis-clos (la plaine de Larchamp est une bande de sable fermée d'un côté par la mer, de l'autre par les hauteurs qui la bordent) ou donne des détails symboliques pour la mort de Vivien, tout comme dans Aliscans (voir les laisses déjà citées à ce sujet).

21 François SUARD: «Chansons de geste et mises en prose: développement du récit d'enfances », dans Perspectives médiévales, n³, octobre 1977, p. 71. 
personnages 》 n'était pas encore accomplie. L'histoire de Vivien reste à inventer dans les Enfances, et elle est construite en écho avec celle de Rainouart (en particulier celui d'Aliscans, qui possède déjà une biographie très personnelle, à caractère romanesque). La vie de Vivien est donc élaborée de manière tout à fait individuelle, dans le souci de construire une destinée particulière en réponse à une autre destinée particulière. Cela souligne le potentiel romanesque du jeune Aymeride qui devient une caractéristique de son personnage qui le différencie des autres héros épiques, et surtout de celui qu'il était lui-même auparavant.

La fenêtre n'est-elle pas la frontière qui marque cette séparation? Vivien n'est plus le même héros et le portrait qui nous est fait de lui à l'occasion de cet épisode s'en ressent. Jusque-là, les portraits de Vivien étaient plus que sommaires. Or, ici, il n'en est rien et l'on peut y lire l'influence du roman:

le portrait dans la chanson de geste est court, surtout si nous le comparons aux développements qu'il connait dans le roman courtois. Il ne répond pas aux définitions édictées par les auteurs des différents arts poétiques de la seconde moitié du XIlème siècle. Il a pourtant tendance à s'amplifier dans les chansons plus tardives. Peut-être estce là l'influence des romans antiques et des premiers romans courtois ${ }^{22}$.

De même, jusqu'alors, ses portraits étaient toujours en rapport avec l'action ou une caractéristique épique du personnage (par exemple, sa blancheur est d'habitude mise en relation avec son caractère de saint-chevalier ${ }^{23}$ ). Ici, l'utilisation de topoï romanesques (les cheveux blonds et bouclés, les yeux vairs comparés à ceux d'un faucon, la délicatesse de la physionomie...) donnent une toute autre image du héros.

On sait en outre les rapports étroits qui unissent le portrait et la description, et l'on a vu à quel point le décor vu de la fenêtre a tout du locus amoenus romanesque. Le portrait et le décor se font face en miroir, avec pour axe de symétrie la fenêtre dans la fiction, et la position dans le mètre de cette même fenêtre au vers 911 (Londres, British Library, Royal 20 DXI).

Le paysage reflet du personnage est en soi, également, un thème éminemment romanesque. On remarquera que ce paysage contient des connotations courtoises qui montrent le héros comme un être lui-même courtois, qui aime se deporter avec son faucon. Il n'est pas inutile d'ailleurs de noter que la transformation de la fenestre en fontaine dans le manuscrit Paris, B.N. fr. 1448, n'est pas si fortuite qu'elle ne le paraît : la fontaine, miroir naturel qui favorise le retour sur soi-même, est le lieu par excellence de la chevalerie romanesque et courtoise et, dans notre texte, la fenêtre le devient à son tour. On y sent toute l'influence de l'idéal courtois, plus tourné vers le loisir, le raffinement des moeurs et le bonheur individuel que l'idéal épique. Il était donc important que la vue sur laquelle donne la fenêtre soit une reverdie et que ce motif courtois apparaisse dans un contexte réflexif. Elle

\footnotetext{
${ }^{22}$ A. MOROLDO : «Le portrait dans la chanson de geste (suite et fin) », dans Le Moyen Age $\mathrm{n}^{\circ} 1$, tome LXXXVII (4 ${ }^{\text {ème }}$ série tome XXXVI), 1981, p. 28 -29.

${ }^{23}$ Nous renvoyons notamment aux laisses déjà citées d'Aliscans.
} 
montre que le monde de l'épopée et celui de la courtoisie sont en train de se rejoindre dans ce personnage éminemment épique qu'est au départ Vivien. La fenêtre est ce par quoi le chevalier épique regarde vers le chevalier courtois, vers une autre sorte d'idéal qui correspond au goût du XIIlème siècle débutant. Cette image, c'est celle de la littérature qui se contemple, qui se réfléchit, au sens physique comme intellectuel du terme, et constate les modifications du paysage épique.

Il semble en effet que l'on puisse lire ce passage comme une réflexion sur le statut de l'épopée, sur les changements dont elle fait l'objet à l'époque mais aussi sur la nécessité de ces transformations. Les Enfances Vivien ont été composées au début du XIII ${ }^{\text {ème }}$ siècle, au moment où le succès du roman est un fait acquis. Comme on l'a vu avec le héros éponyme, l'oeuvre subit incontestablement l'influence de ce genre à la mode, de même que la chanson de geste en général ${ }^{24}$. Dès lors, cette fenêtre par laquelle le héros regarde au loin est peut-être métaphorique d'un seuil dans l'histoire littéraire du Moyen Age dont l'auteur des Enfances paraissait tout à fait sentir l'importance. Il n'est donc pas anodin qu'un Aymeride aille à la fenêtre pour deporter et chanter: cela signifie la volonté de cette chanson de se tourner vers un lyrisme et une littérature purement plaisantes, comme le roman avait la réputation de l'être. En outre, on a dit que le verbe se deporter était une référence à l'idéal courtois du bonheur personnel. Il participe du même mouvement d'intérêt porté à l'individu que la mélancolie et le retour sur soi provoqués par la fenêtre et son paysage, mouvement qui s'oppose bien entendu à la vocation d'histoire collective de l'épopée. Chez Mabile, toute l'attention est portée sur Vivien en tant qu'individu, de même que choisir d'écrire un récit d'enfance, c'est s'intéresser à un personnage et à son développement personnel. On n'est même plus dans la « différenciation» des personnages dont parlait François Suard, mais plutôt dans une volonté d'individualisation, ce qui est beaucoup plus radical. Cet intérêt porté à l'individu induit des différences de traitement dans le récit : ainsi, la place accordée à l'analyse des sentiments est plus grande, et l'on peut trouver des nuances plus fines dans la palette des émotions, comme on le voit ici avec la nostalgie.

Peut-être l'auteur regrette-t-il cette évolution et la déploration de Vivien sur sa parenté héroïque est-elle aussi celle de l'auteur sur la grande époque de la chanson de geste et du héros épique. Pour autant, il nous présente ce changement comme nécessaire: le paysage qu'observe le personnage est un paysage de reverdie, un paysage fertile en opposition avec les vastes plaines arides et desséchées de

\footnotetext{
${ }^{24}$ «Après 1150, en même temps que le genre prolifêre [...] et se développe de manière homogène, des interférences se produisent avec le roman, dans le ton et les thèmes. La distinction des deux genres tend à devenir purement technique : la chanson de geste continue à être chantée et est écrite en laisses »(Paul ZUMTHOR : Histoire littéraire de la France médiévale (VI ${ }^{\text {ème }}$-XIV ${ }^{\text {ème }}$ siècles), Paris, PUF, 1954, p. 142-143). Il n'y a qu'à observer une oeuvre comme Folque de Candie ${ }^{24}$, probablement de peu postérieure aux Enfances Vivien, pour constater que la frontière entre chanson de geste et roman, même dans un cycle aussi traditionnellement épique que celui de Guillaume d'Orange, est devenue plus que ténue.
} 
Larchamp. La seule ouverture possible vers l'avenir et le renouveau, pour la chanson de geste, est de se tourner vers le roman et d'exploiter les potentialités romanesques de grands héros épiques tels que Vivien. La fenêtre est un espace ouvert sur le monde, elle donne l'occasion du chant. Le monde est la grande voix lyrique qui inspire Vivien et chante à travers lui : ce monde, c'est la voix de la chanson de geste, de toute la geste française convoquée à travers les noms des héros parents de Vivien, mais aussi la voix du lyrisme romanesque à travers le monde du roman mis en abyme à travers son décor le plus typique, le locus amoenus. C'est l'invention d'un lyrisme mixte. Vivien regrette l'ancienne épopée et pleure sur les héros du passé, mais il réussit quand même une synthèse, comme le montrent les paroles des marchands qui reconnaissent son appartenance à la geste en même temps qu'ils le perçoivent dans un contexte romanesque. Vivien se tient à la fenêtre, au seuil, à l'image de ce que devient, et peut-être doit devenir pour survivre, la chanson de geste.

Ainsi le rôle de la fenêtre dans notre passage est-il toujours en tension dans un double mouvement: par sa présence en tant qu'objet, elle modifie le traitement du personnage et par là-même les modes du récit ; par sa fonction de métaphore, elle est la représentation sensible de toutes ces modifications. Elle révèle l'entre-deux dans lequel Vivien est plongé par sa condition sociale singulière, mais elle montre surtout sa véritable nature héroïque et chevaleresque. Dès lors, elle n'est plus seulement une fenêtre ouverte sur l'intériorité du héros mais sur tous les possibles du récit qu'elle relance efficacement. Elle s'ouvre sur l'essence du personnage, mais aussi sur son destin et surtout sur son potentiel romanesque. Car la fenêtre est un indicateur, voire un instrument, de la réflexivité du récit. C'est moins Vivien qu'elle nous montre que le clerc, un homme de littérature qui s'interroge sur son art, qui voit l'épopée évoluer et veut en traduire la métamorphose : que ce soit la formation de cycles nouveaux par ramifications à l'intérieur d'une geste ou l'absorption d'un genre par un autre, la fenêtre reflète tous ces mouvements et toutes les réflexions sur un genre encore en transformation malgré son ancienneté. L'auteur a conscience de cette hybridation et la traduit en image : la fenêtre s'ouvre sur l'avenir littéraire et son infinie capacité à se renouveler, son éternelle reverdie. 